\title{
Combien ou comment? \\ Les femmes canadiennes dans les récits scolaires et dans la mémoire collective, rétrospective des recherches depuis 1980
}

\author{
Marie-Hélène Brunet \\ Université d'Ottawa
}

\section{RÉSUME}

L'histoire des femmes peine toujours à faire sa place dans la mémoire collective et dans l'enseignement de l'histoire au Canada. Le présent article propose une courte recension des écrits publiés depuis 1980 à ce sujet. Si certaines recherches ont posé la question à savoir " combien " de femmes étaient présentes dans les récits, c’est surtout le "comment " qui a intéressé les chercheuses et les chercheurs. Les études, provenant des départements d'histoire et des facultés d'éducation, ont porté sur trois aspects : la mémoire collective, les manuels scolaires et la compréhension des élèves du rôle des femmes dans l'histoire. Malgré la très lente évolution de la représentation des femmes dans la trame narrative de l'histoire canadienne, l'analyse des recherches sur cette question permet un regard critique sur la construction des récits historiques et mène à une réflexion nécessaire sur les événements commémoratifs récents.

\begin{abstract}
Women's history is still waiting to gain more place in collective memory and history teaching in Canada. This article provides a brief review of the literature published since 1980 on this topic. While some research has posed the question of "how many" women are present in the narratives, "how they are represented" was the focus of most researchers. Studies from history departments and faculties of education concentrated on three aspects: collective memory, textbooks, and student understanding of women's agency. Despite the very slow evolution of the representation of women in the narrative framework of Canadian history, this paper allows a critical perspective at the construction of historical narratives and leads to a necessary closer look on recent commemorative events.
\end{abstract}

\section{Introduction}

En 2016, le Conseil du statut de la femme publiait au Québec une étude relevant une fois de plus la faible proportion et la représentation stéréotypée des femmes dans les manuels et les programmes d'histoire. ${ }^{1}$ Ce constat semble d'autant plus décourageant 
qu'il differe peu des conclusions de recherches datant déjà de plusieurs décennies, au Québec, mais aussi ailleurs au Canada et dans le monde. Ainsi, l'inclusion des femmes dans les récits historiques étudiés à l'école se fait à pas de tortue, et ce, malgré les développements fulgurants dans les départements d'histoire en histoire des femmes et du genre. La transposition didactique est un processus complexe, et plusieurs facteurs, surtout de nature politique, expliquent la lenteur de l'évolution. Le présent article dresse un portrait général des recherches actuelles et passées sur les femmes dans les récits scolaires et de mémoire collective au Canada, tout en faisant référence à certaines recherches débordant de ce cadre géographique. Ce bilan s'appuie sur des études provenant des départements d'histoire comme des départements d'éducation, mais adopte une perspective historique. De surcroît, il élargit l'examen de ces enjeux au-delà des frontières disciplinaires et porte un regard vers le futur : quel avenir pour les questions de genre autant en recherche que dans les programmes scolaires?

L'année 2017 en a été une de commémoration : 150 ans de la Confédération, 375 ans de la fondation de la ville de Montréal. Or, ces célébrations créent des heurts par leur caractère trop souvent exclusif, la mémoire collective étant un terrain de contestation. ${ }^{2}$ Les multiples activités de commémoration visent d'ailleurs très souvent à attiser des sentiments d'appartenance à une histoire commune. Évidemment, une telle entreprise, sans être nécessairement vouée à l'échec, attire son lot de critiques. Il n'y a qu’à penser ici à la polémique engendrée par la diffusion de la série télévisée The Story of $U s^{3}{ }^{3}$ Si l'on peut arguer que les célébrations de la mémoire collective sont différentes de l'histoire enseignée — du moins on ose l'espérer —, il n'en reste pas moins qu'elles frayent leur chemin dans les salles de classe et font partie de l'expérience scolaire et extrascolaire des élèves (et de la population en générale); que ce soit au travers de visites muséales, de trousses pédagogiques et autres produits dérivés destinés au public scolaire. ${ }^{4}$ Qui plus est, malgré les finalités critiques de la majorité des programmes d'histoire à l'échelle canadienne, les contenus prescrits, de même que les pratiques pédagogiques réelles, démontrent que la mémoire collective influence toujours de façon notable l'histoire scolaire. ${ }^{5}$

Qu'en est-il des femmes qui sont célébrées dans cette mémoire ou étudiées dans les écoles? Si la question du " combien » est légitime, considérant que la moitié de la population est de sexe féminin, elle n'est certainement pas suffisante. Plus encore, c'est en se tournant vers le " comment " que les recherches aident à comprendre la représentation des femmes dans les récits historiques. Ainsi, certaines femmes ont été jugées porteuses d'une symbolique historique marquante, alors que d'autres ont été écartées; ces choix ne sont pas sans conséquence. Les chercheuses et chercheurs ont aussi interrogé la progression, s'il en est une, de la représentation des femmes dans les programmes scolaires depuis les dernières décennies et analysé les changements à l'aune du contexte social et des réformes pédagogiques. La grille d'analyse basée sur le concept de genre a permis une compréhension accrue des forces en présence.

Certaines recherches en éducation éclairent aussi d'autres aspects tout aussi intéressants et importants reliés à des aspects plus pédagogiques comme la compréhension de l'histoire des femmes par les élèves ou leurs enseignants. Elles donnent ainsi des 
pistes à savoir « comment " réfléchir de façon critique aux représentations des femmes dans la mémoire collective, dans les programmes et dans les manuels scolaires.

C'est donc à travers une approche multidisciplinaire (histoire des femmes, histoire de l'éducation, didactique de l'histoire, genre et éducation) que nous abordons le sujet. Dans un contexte où les commémorations se multiplient et occupent un espace médiatique et social prépondérant, il semble justifié d'offrir une synthèse de recherches variées ayant interrogé de manière critique les liens entre genre, histoire scolaire et mémoire collective.

Par souci de concision, nous nous limiterons ici à des recherches publiées depuis 1980. Cette balise chronologique touche toutefois uniquement les études choisies et non l'analyse historique en elle-même puisque certaines recherches se sont intéressées à l'évolution de la représentation des femmes depuis le $19^{\mathrm{e}}$ siècle. Nous ne prétendons pas ici à une présentation exhaustive de toutes les recherches ayant touché le sujet,

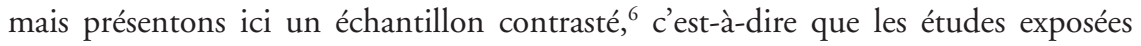
dans le cadre de l'article apportent des éléments significatifs, présentent une approche singulière ou novatrice ou encore, permettent une analyse plus diversifiée au niveau géographique. À quelques reprises, des références supplémentaires sont suggérées en notes de bas de page.

\section{Le grand récit national : quelle place pour les femmes?}

L'historienne et didacticienne Micheline Dumont sait trouver les mots justes pour dénoncer avec précision l'exclusion des femmes du récit historique :

L'histoire écrite n'a jamais tenu compte de l'ensemble de la réalité. Elle a été la création d'une minorité qui s'en est servie pour justifier et imposer sa domination. Ce que les femmes ont fait et dit a été laissé de côté et jugé insignifiant. L'histoire s'est donc avérée partielle : elle a oublié le plus souvent les femmes. Elle est aussi partiale : elle n'a présenté que le point de vue des hommes, tout en le considérant comme objectif et universel. Posé comme sujet, le masculin a construit sa vision du monde. Défini comme objet, le féminin a été modulé selon les critères changeants du savoir masculin. ${ }^{7}$

Pour Dumont, c'est une véritable révolution qu'il faut entreprendre pour déconstruire des paradigmes d'écriture et de pensées scientifiques qui reproduisent, encore aujourd'hui, la quasi-absence des femmes dans les récits historiques, particulièrement dans les grandes synthèses historiques et dans les programmes scolaires. ${ }^{8}$

Cette révolution est déjà entamée si l'on se fie aux recherches foisonnantes des dernières décennies dans les départements d'histoire du pays; elle l'est toutefois moins lorsqu'on s'intéresse à l'histoire scolaire ou publique. La mémoire collective, plus difficile à circonscrire ou à analyser comme objet, a néanmoins été analysée au travers de multiples sources, notamment culturelles. Cette mémoire n'est évidemment pas fixe à travers le temps — ou dans l'espace dans un pays aux identités multiples comme le Canada - , mais le fait que plusieurs personnages ou événements conservent un 
statut quasi mythique au fil des siècles et des décennies a amené des historiennes et des historiens à les examiner plus en profondeur.

Grâce à une grille d'analyse basée sur le genre, concept popularisé en histoire par Scott et s'intéressant aux constructions sociales et historiques de la catégorisation du masculin et du féminin, plusieurs historiennes et historiens se sont interrogés sur les représentations genrées, symboliques ou tangibles, que l'on retrouve dans la mémoire collective. ${ }^{9}$ En 2002, Coates et Morgan proposent une analyse des représentations de Laura Secord et de Madeleine de Verchères dans les mémoires collectives canadiennes. L'examen est fascinant et révèle dans les deux cas une construction genrée et racialisée de la mémoire collective (la femme héroïne porteuse de la vertu - l'Iroquois incarnant au contraire la représentation de l'homme cruel). Les motivations et, plus globalement, les vies de ces deux femmes ne sont jamais considérées au-delà de l'événement ponctuel qui les a fait connaître (Guerre de 1812, attaque du FortVerchères en 1692). ${ }^{10}$

En 2012, le mémoire de Fortin s'intéresse aux représentations des pionniers et pionnières de la Nouvelle-France dans la mémoire collective canadienne-française et québécoise. La représentation des femmes, notamment des filles du roi, par les historiens des $19^{\mathrm{e}}$ et $20^{\mathrm{e}}$ siècles sert ici à assoir les valeurs morales de la famille canadiennefrançaise dans laquelle le statut de la " mère » est particulièrement important pour la pérennité de l'identité et la cohésion nationale. ${ }^{11}$

L'analyse des symboliques genrées est poussée encore plus loin par Lanthier en 1998, qui, par l'analyse de discours et d'éléments culturels associés au nationalisme radical des années 1960 et 1970, vient démontrer la puissance de la violence envers les femmes dans les récits où la symbolique femme-pays et la persistance de mythes (par exemple la mère toute puissante et castratrice) ont pour conséquence de discréditer et d'exclure la parole des femmes, incluant celles adhérant au mouvement nationaliste. ${ }^{12}$

Dans la mémoire collective donc, il semble que « les femmes sont élevées au rang de déesses [ou de symboles,] mais ne sont pas tolérées dans la discussion ou l'action ${ }^{13}$ Est-ce bien différent dans les manuels et les programmes scolaires?

\section{Intruses ou incluses? Les femmes dans les manuels et programmes d'histoire}

Une grande part des recherches sur l'intégration des femmes à la trame narrative historique étudiée dans les écoles s'est concentrée sur l'analyse des manuels. Notons d'abord que ces études souffrent d'une limite, soit celle d'être détachée de l'expérience en classe et de l'usage réel, par les élèves et par les enseignants, de cet outil didactique. Néanmoins, ces recherches sont nécessaires et ont servi, au fil du temps, à dénoncer la quasi-absence des femmes dans l'histoire enseignée ou les stéréotypes que celle-ci perpétue au travers de choix narratifs.

À ce sujet, le travail de Tetreault, aux États-Unis, fait parfois office de texte fondateur, même s'il se base sur les travaux d'historiennes des décennies précédentes. L'auteure propose ainsi cinq phases d'intégration de l'histoire des femmes dans les programmes et manuels scolaires; cette classification a par la suite été reprise par 
d'autres recherches dans le domaine. Au plus bas niveau, on dénote l'absence totale des femmes dans le récit du manuel. Vient ensuite l'histoire compensatoire : les femmes d'exception, celles qui ont été actives dans la " cour des grands ", le plus souvent au niveau politique ou scientifique, font alors leur apparition dans le récit, à titre individuel — et souvent « héroïque ». Le troisième niveau, celui de la " contribution " inclut les femmes ayant participé à de grands événements : les guerres, les luttes syndicales ou même certaines luttes féministes (surtout le droit de vote); la contribution est alors individuelle ou collective, mais très souvent sans nuance (pas de mention des oppositions, des expériences variées des femmes, des luttes dans la durée). L'objet de recherche de la majorité des historiennes et historiens du genre et des femmes, soit des aspects historiques souvent négligés dans les récits traditionnels (vie quotidienne, sexualité, travail domestique, travail salariée des femmes, présence des femmes dans les sphères culturelles et intellectuelles, etc.), arrive au quatrième niveau. Finalement, ce n'est qu'au dernier niveau que l'intégration est réelle. En d'autres mots, la séparation des genres n'est pas visible dans la mise en page physique du texte du manuel. Il n'y a pas d'ajouts de paragraphe ou de section spéciale dédiée aux femmes de façon isolée, puisque les femmes font intégralement partie du récit central. L'histoire mixte ou la " nouvelle histoire universelle » ainsi constituée est celle souhaitée, un souhait qui demeure lettre morte dans les manuels analysés par Tétreault qui se classent tous au deuxième ou troisième niveau, ne dépassant jamais le stade de la contribution. ${ }^{14}$

Des études canadiennes ont aussi cherché à quantifier, et parfois à qualifier, la présence des femmes dans les manuels et les programmes d'histoire. Par l'analyse de manuels publiés depuis les années 1950 en Colombie-Britannique, Clark dresse un parallèle entre le "nation-building " et la marginalisation des femmes dans les textes scolaires. Ainsi, la narration d'une grande épopée visant avant tout à aviver les ferveurs patriotiques des jeunes Canadiens a eu pour effet de centrer le propos sur des événements politiques fondateurs et des grands personnages, écartant d'emblée les figures féminines. Elle constate que ce n'est qu'à partir des années 1980 que celles-ci font leur apparition, mais uniquement de manière contributoire, çà et là, sans intégrer réellement la trame narrative centrale qui demeure étroitement liée à la mémoire collective. ${ }^{15}$

Au Québec, Efthymiou s'est penchée sur l'évolution de la présence des femmes dans les manuels publiés entre 1980 et 2004. Elle a comptabilisé les «notations genrées ", c'est-à-dire des notions historiques généralement associées aux hommes (ex. : les instances politiques en Nouvelle-France), aux femmes (ex. : l'éducation des enfants à la maison), ou neutres (ex. : « la population »). Ce sont les notations masculines qui occupent la plus grande proportion du texte (60\%) alors que les notations associées aux femmes ne dépassent pas $8 \%$; le reste étant des notations « neutres ». Par ailleurs, elle décèle une " compartimentation » des événements relatifs à l'histoire des femmes qui prend deux formes. D'une part, les femmes sont souvent reléguées aux encadrés extérieurs au texte central de même qu'aux documents iconographiques. D'autre part, l'histoire des femmes peut être facilement isolée dans les manuels puisque certaines pages sont dédiées spécifiquement à ce sujet. À l'extérieur de ces pages, les femmes disparaissent totalement du récit. ${ }^{16}$ Cette constatation est commune à presque toutes 
les études consultées. Ainsi, nous notons que dans la progression des apprentissages publiée en 2011 pour le cours d'histoire et d'éducation à la citoyenneté du deuxième cycle du secondaire, ${ }^{17}$ aucun élément de connaissance à maitriser ne faisait référence aux femmes pour toute la période allant de la fin de la Nouvelle-France à la Crise économique, soit une période de près de 170 ans sans aucune mention du rôle historique des femmes. ${ }^{18}$ Cette absence semble assez commune dans les grands récits de l'histoire nationale au Québec et au Canada. Une visite dans la Salle de l'histoire canadienne du Musée canadien de l'histoire, une exposition d'ailleurs complètement renouvelée pour l'anniversaire de la Confédération, laisse aussi cette impression. Si les femmes font leur apparition dans la première et dans la dernière salle; elles sont très difficiles à trouver dans la seconde partie de l'exposition, celle s'intéressant au Régime britannique. Il semble ainsi que l'importance accordée aux événements militaires et politiques de cette période dans la mémoire collective et dans les programmes scolaires tend à exclure le rôle important des femmes de cette époque dans l'évolution du Canada.

En Ontario, Cosantino a proposé une analyse de manuels publiés dans la première décennie du $21^{\mathrm{e}}$ siècle et considérés comme conformes au curriculum de cette province. Les femmes sont présentes dans environ $5 \%$ du texte (proportion du nombre de pages où au moins une femme est nommée sur le nombre de pages total) et se retrouvent dans $16 \%$ des documents iconographiques. La chercheuse ajoute que, lorsque l'on regarde plus en détail le choix des personnages féminins, un problème supplémentaire surgit : elles sont presque toutes blanches et proviennent de l'élite. C'est donc dire qu'aucune mention n'est faite des discriminations intersectionnelles dont les femmes de groupes minorisés ont été victimes dans l'histoire canadienne. ${ }^{19}$

D'autres recherches se sont aussi intéressées au contexte canadien ou plus spécifiquement à une province; les conclusions sont toujours assez similaires aux études présentées ci-haut. ${ }^{20}$ Certaines recherches venant de la France apportent selon nous des éléments originaux, notamment parce qu'ils adoptent clairement le genre comme grille d'analyse.

Les observations de Mang sur les manuels scolaires utilisés dans les lycées français dans les années 1980 et 1990 abordent de nouveaux aspects dépassant la comptabilisation..$^{21}$ Les discontinuités du récit illustrent de façon patente la marginalisation des femmes; reléguées aux documents externes (surtout iconographiques) ou à des dossiers connexes. Qui plus est, la conception visuelle de ces dossiers ou documents se rapproche souvent de celles des magazines féminins - jusqu'au choix des couleurs, surtout roses, rouges et mauves - ce qui tend à réduire la crédibilité de ces pages, au profit du texte régulier et de son aspect plus « sérieux ». En somme, la présentation des femmes dans les manuels se limite donc à un « niveau anecdotique et aproblématique $"{ }^{22}$ L'exemple suivant reflète ces deux limites :

Les femmes descendent dans la rue, est-il précisé, à l'occasion de la « journée internationale de la femme ». Mais bien des questions restent en suspens : quelle est l'origine et la portée de cette journée? Quelle a été la motivation des femmes ce jour-là? Sont-elles de simples ménagères en colère ou des citoyennes 
mobilisées par les mots d'ordre révolutionnaires? Quel a été l'impact concret de leur action dans la rue auprès des forces de l'ordre et de leurs frères, compagnons ou maris? ${ }^{23}$

Puisque les femmes occupent une part plus importante-mais, répétons-le, bien en deçà d'une proportion représentative — des éléments iconographiques, il est intéressant de se tourner vers le choix des images. Mang note que les manuels français valorisent deux catégories d'images : celles dans lesquelles les femmes sont porteuses d'une symbolique, telles des allégories de la République (comme Marianne), puis les représentations artistiques (peinture, sculpture) ou publicitaires (affiches de propagande, publicités). Or, ces deux catégories sont probablement celles qui laissent le moins de place aux femmes comme agentes de l'histoire. Bien au contraire, elles reflètent, même inconsciemment, les structures patriarcales. Notons aussi que les manuels, en ne proposant pas de problématisation par rapport à ces images, ne peuvent favoriser une réflexion critique. Un peu moins de 20 ans plus tard, une étude française s'intéressant à cette même question avec des manuels plus récents réitère ces résultats, attestant une fois de plus de la lenteur de l'évolution. ${ }^{24}$

Une recherche historique est en cours en ce moment, tant au niveau iconographique qu'au niveau du texte, pour les manuels québécois d'histoire publiés entre 1954 et 1980. Bien qu'encore à ses balbutiements, l'enquête de Clapperton-Richard adopte aussi une perspective genrée qui démontre que les femmes se retrouvant dans les récits correspondent à un idéal de féminité; une écriture qui perdure dans les récits scolaires au travers du temps et qui traduit une stagnation dans la représentation des femmes dans l'écriture de l'histoire. ${ }^{25}$

En Ontario, dans sa thèse déposée en 2012, Rose Fine-Meyer apporte une contribution originale à la question des mécanismes par lesquels l'histoire des femmes a pu faire son chemin dans les écoles ontariennes entre 1968 et 1993. Si le ministère de l'éducation ontarien s'est montré ouvert aux changements, ce sont plutôt les initiatives personnelles d'enseignantes et d'enseignants, de même que le travail acharné de groupes féministes qui ont permis une certaine évolution. C'est cependant en marge du curriculum, au travers par exemple de ressources pédagogiques originales créées localement ou par des organismes externes au ministère, que des élèves ontariens ont pu découvrir certains aspects de l'expérience historique des femmes. ${ }^{26}$

Plusieurs angles demeurent à analyser quant à la présence et à la représentation des femmes dans les récits scolaires. Il serait intéressant par exemple de considérer la comparaison des programmes des différentes provinces à travers le temps. Au Québec et ailleurs, les programmes d'histoire sont souvent critiqués; ils sont modifiés à intervalles relativement réguliers. ${ }^{27}$ On ne peut cacher ici la part importante jouée par les motivations politiques de certains acteurs. Au Québec, entre autres, les débats sur l'enseignement de l'histoire ne sont pas sans rappeler l'importance de la question nationale dans l'espace public et médiatique. Or, la question de la place des femmes n'est que rarement évoquée dans ces débats et nous constatons, avec désarroi, que cela n'aide certainement pas à renouveler l'écriture des programmes vers une meilleure intégration de l'histoire des femmes. 


\section{Les élèves, leurs enseignants et l'histoire des femmes}

Au-delà des programmes et des manuels se trouvent des éléments non négligeables à la compréhension de l'intégration des femmes en classe d'histoire. D'abord, le récit des enseignants n'est pas qu'un simple calque des exigences ministérielles ou du matériel didactique. Mais encore plus, il faut se demander comment les élèves interagissent avec les savoirs enseignés — ou l'absence de ceux-ci — en lien avec l'histoire des femmes?

Ce champ de recherche s'intéressant davantage aux idées des élèves et de leurs enseignants sur l'histoire et les contenus scolaires est relativement récent, mais il en dit long, comme nous le verrons, sur la manière dont l'enseignement de l'histoire forge des représentations coriaces et encourage la reproduction d'une trame historique se rapprochant de la mémoire collective.

Une recherche en cours à l'Université de Sherbrooke vise à analyser les représentations des enseignants et des élèves par rapport à certaines luttes sociales dans l'histoire. Les chercheuses ont observé des classes, rencontré des enseignants, et analysé le matériel et les évaluations distribuées en classe relativement à l'histoire du féminisme. ${ }^{28}$ Leurs constats recoupent la récente étude de Scheiner-Fisher en Floride dans laquelle plus de la moitié des enseignants déclare intégrer la voix des femmes à leur leçon moins d'une fois par mois. Le manque de temps, l'aspect "non obligatoire " du curriculum et leur propre méconnaissance de l'histoire des femmes figurent parmi les raisons évoquées pour expliquer la situation. Même les leçons observées en classe par les chercheurs encourageaient la perpétuation des stéréotypes de genre ou l'idée que l'égalité était désormais acquise. ${ }^{29}$ Somme toute, il apparaît primordial que la formation des maitres en histoire fasse davantage de place au genre comme catégorie d'analyse de même qu'à une réflexion sur la construction des savoirs historiques.

Si l'on se tourne vers l'autre côté de la classe, des études récentes canadiennes et états-uniennes témoignent d'une compréhension téléologique de l'histoire des femmes, ou du moins de l'histoire du féminisme, chez les élèves. Il faut noter ici que les programmes prescrits, en se limitant à une histoire contributoire, limitent l'histoire des femmes — du moins pour le $20^{\mathrm{e}}$ siècle — à une histoire des mouvements féministes (et, le plus souvent, de façon assez réductrice). Par conséquent, l'interprétation des élèves est souvent celle d'une " histoire terminée " liée à une "égalité désormais acquise ». Levstik et Groth, ayant expérimenté un enseignement de l'histoire incluant plus de femmes que le curriculum traditionnel, soulignent que les élèves de sexe féminin, malgré un intérêt perceptible pour la matière renouvelée, ont perçu cette modification du cours comme étant préjudiciable aux garçons. Les jeunes ayant participé à l'étude considèrent que l'histoire des femmes ne fait pas partie de l'histoire " normale $»{ }^{30}$ Cette attitude s'observe aussi chez certains enseignants qui déclarent qu'il " n'y a pas tant de femmes dans l'histoire ". ${ }^{31}$

Des recherches récentes amènent des éléments prometteurs pour le futur. Le travail d'analyse critique, à partir de documents iconographiques présentant des femmes, ou encore en partant de la comparaison des récits de manuels, peut amener les élèves à développer leur compréhension du rôle actif des femmes dans l'histoire. ${ }^{32}$ Ces études 
permettent d'approcher la quasi-absence des femmes et leur représentation biaisée, non comme un obstacle, mais comme une possibilité. Ainsi, s'il demeure légitime et indispensable de continuer à dénoncer l'iniquité des programmes et des manuels, il faut aussi réfléchir aux aspects pédagogiques qui permettent de porter un regard critique devant un contenu androcentrique.

\section{Conclusion}

Trois catégories de recherches ont été exposées dans cet article. D’abord, celles s'étant intéressées aux figures féminines et aux représentations de la féminité dans la mémoire collective. Ensuite, celles s'étant penchées sur l'analyse des manuels et des programmes d'histoire, passés et présents, en fonction de la place occupée par l'histoire des femmes. Finalement, nous avons ouvert la discussion sur un champ de recherche moins développé, mais prometteur, soit celui des représentations des élèves et de leurs enseignants par rapport à l'histoire des femmes. Chacune de ces catégories a contribué de façon importante à mieux comprendre l'évolution de la présence des femmes dans les récits scolaires et dans la mémoire collective.

Après la lecture de ce texte, nombreux sont ceux qui concluraient à une vision défaitiste ou même cynique de l'avenir. Au contraire, nous sommes plutôt optimistes. En effet, la vitalité de la recherche en histoire de l'éducation, en histoire et en didactique de l'histoire, de même que la convergence de ces différents domaines de recherche, nous portent à croire que l'apport historique des femmes ne peut que gagner en visibilité. Assurément, un travail colossal demeure à faire pour comprendre les mécanismes d'évolution de la mémoire collective et de l'enseignement de l'histoire. L'intégration, plus que contributoire, des femmes aux récits historiques populaires ou aux programmes et manuels scolaires, prendra certainement encore plusieurs décennies, surtout si l'on se fie à la lenteur des changements opérés jusqu’à présent. Mais, tout compte fait, la réflexion se multiplie et elle trouve, lentement mais sûrement, son chemin dans l'espace public et médiatique. Nous espérons avoir offert dans cet article une vue d'ensemble synthétique et multidisciplinaire éclairant le potentiel immense des recherches liées à ces questions. En outre, la diffusion des recherches peut contribuer à sensibiliser les enseignants, mais aussi la population en général, à l'importance d'étudier et de comprendre le rôle fondamental qu'ont joué les femmes dans l'histoire.

À la veille des célébrations du centenaire du droit de vote des femmes au niveau fédéral, il apparaît d'autant plus pertinent de porter un regard critique sur la manière dont le gouvernement et d'autres organismes publics décideront de commémorer cette lutte historique. L'histoire de l'éducation, notamment au travers des recherches présentées dans cet article, permet de donner des outils d'analyse pour appréhender ces commémorations. Par exemple, insistera-t-on sur le rôle du gouvernement dans l'octroi du droit de vote aux femmes, ou plutôt sur les luttes féministes, dans la durée, pour l'obtention de ce droit? La différence est importante. Quels personnages (Robert Borden, Mary Ann Schadd, etc.) seront célébrés, mais surtout, " comment " ceux-ci et celles-ci seront-ils présentés? Ces commémorations peuvent-elles jouer un 
rôle dans la diffusion des recherches, beaucoup plus diverses, sur l'histoire des femmes au Canada? À l'opposé, un peu à la manière des manuels scolaires, viendront-elles à nouveau réduire la participation des femmes à l'histoire canadienne à un événement isolé ou pire, réduire les luttes féministes dans l'histoire à la simple célébration d'une victoire ponctuelle?

\section{Autres ouvrages incontournables}

Dumont, Micheline, Le féminisme québécois raconté à Camille (Montréal : Remue-ménage, 2008).

Heap, Ruby et Alison Prentice, dir., Gender and Education in Ontario (Toronto : Scholar's Press, 1991).

\section{Notes}

1 Conseil du statut de la femme, Avis. L'égalité entre les sexes en milieu scolaire (Québec: Gouvernement du Québec, 2016), http://www.csf.gouv.qc.ca/wp-content/uploads/ avis_egalite_entre_sexes_milieu-scolaire.pdf.

2 Les définitions de la mémoire collective sont polysémiques. La distinction entre mémoire et histoire proposée par Joutard nous semble ici la plus en lien avec notre propos. Ainsi, le récit historique «instaure d'entrée de jeu une distance» (p. 15). Les prétentions des programmes scolaires actuels au Canada s'enlignent d'ailleurs dans cette direction avec le développement d'habiletés critiques et de compétences en lien avec la pensée historique. La mémoire, quant à elle, a une dimension émotionnelle et identitaire; elle est selon Joutard " un passé dans le présent ». Philippe Joutard, Histoire et mémoires, conflits et alliance (Paris : La Découverte, 2013).

3 Voir entre autres Jean-François Nadeau, «Série historique. Le producteur défend sa version non « exhaustive » de l'histoire du Canada, Le Devoir, 5 avril 2017.

4 Voir par exemple Alexandre Lanoix, Historica et compagnie, l'enseignement de l'histoire au service de l'unité canadienne, 1867-2007 (Montréal : LUX, 2007); Ken Osborne, "Teaching History in Schools: A Canadian Debate", Curriculum Studies 35, no. 5 (2003).

5 Pour les contenus enseignés, voir Marc-André Éthier, Jean-François Cardin et David Lefrançois, "Cris et chuchotements : la citoyenneté au cœur de l'enseignement de l'histoire au Québec, Revue d'histoire de l'éducation 65, no. 2 (2013). Pour les pratiques pédagogiques, voir Vincent Boutonnet, «Pratiques déclarées d'enseignants d'histoire au secondaire en lien avec leurs usages des ressources didactiques et l'exercice de la méthode historique ", McGill Journal of Education 50, no. 2-3 (2015).

6 Pour en savoir plus sur l'échantillonnage raisonné par contraste, voir Alvaro Pires, "Échantillonage et recherche qualitative : essai théorique et méthodologique " dans Jean Poupart, Jean-Pierre Deslauriers, Lionel-Henri Groulx, Anne Laperrière, Robert Mayer et Alvaro Pires, dirs, La recherche qualitative : enjeux épistémologiques et méthodologiques (Boucherville : Gaëtan Morin éditeur, 1997), 113-169.

7 Micheline Dumont, "La construction de l'invisibilité ", Revue Liberté 42, no. 4 (2000) : 12.

8 Voir par exemple la critique de Micheline Dumont de l'ouvrage de synthèse Histoire du Québec pour les nuls d'Éric Bédard : Micheline Dumont, "Au sujet de l'Histoire du Québec pour les nuls, Les femmes ne sont nulle part! ", Le Devoir, 21 décembre 2012. 
9 Joan W. Scott, "Gender : A Useful Category of Historical Analysis ", The American Historical Review 91, no. 5 (1986) : 1054.

10 Colin M. Coates et Cecilia Morgan, Heroines and History : Madeleine de Verchères and Laura Secord (Toronto : University of Toronto Press, 2002).

11 Marie Fortin, La représentation des pionniers et des pionnières dans les récits sur les origines nationales au Canada français, mémoire de maîtrise, Université de Montréal, 2011.

12 Stéphanie Lanthier, L'impossible réciprocité des rapports politiques et idéologiques entre le nationalisme radical et le féminisme radical au Québec, 1961-1972, mémoire de maîtrise, Université de Sherbrooke, 1998.

13 Micheline Dumont, "La construction de l'invisibilité historique des femmes dans l'histoire ", conférence donnée le 26 septembre 2016 dans le cadre de la rentrée universitaire, Université de Sherbrooke, APPRUS, 9.

14 Mary Kay Thompson Tetreault, «Integrating Women's History : The Case of United States History High School Textbooks ", History Teacher 19 (1986) : 212.

15 Penney Clark, " "A Nice Little Wife to Make Things Pleasant" : Portrayals of Women in Canadian History Textbooks Approved in British Columbia ", McGill Journal of Education 40, no. 2 (2005) : 244-245.

16 Loukia Efthymiou, "Sexe, genre et histoire : visibilité des sexes et représentation des genres dans les manuels d'histoire francophones du secondaire québécois, 1980-2004 ", dans Nicole Lucas, dir., Femmes et genre dans l'enseignement (Paris : Le Manuscrit, 2007), 45-70.

17 Ministère de l'éducation, du loisir et du sport, Progression des apprentissages au secondaire: Histoire et éducation à la citoyenneté $3^{e}$ et $4^{e}$ secondaire (Québec : Gouvernement du Québec, 2011).

18 Marie-Hélène Brunet, "Une histoire sans les femmes est une histoire désengagée ", Histoireengagée.ca, 2013, http://histoireengagee.ca/wp-content/uploads/2013/11/ BRUNET-Marie-Hélène.-Une-histoire-sans...-1.pdf

19 Christine Danielle Cosentino, The Treatment of Women's History in the Grade 10 Ontario Curriculum and Selected Textbooks, mémoire de maîtrise, Mount Saint Vincent University, 2008.

20 Voir par exemple Patricia Baldwin et Douglas Baldwin, "The Portrayal of Women in Classroom Textbooks ", Canadian Social Studies 26, no. 3 (1992) : 110-114; ou encore Rosemarie Brodeur, "Un homme sur deux est une femme ". Intégrer le genre dans les manuels d'histoire de la civilisation occidentale au collégial : quelques propositions, mémoire de maîtrise, Université de Montréal, 2011.

21 Philippe Mang, "Les manuels scolaires ont-ils un genre? ", dans Michel de Manassein, dir., De l'égalité des sexes (Paris : CNDP, 1995), 279-292.

22 Ibid., 285.

23 Ibid., 284.

24 Alice Stephan, Les femmes et leurs représentations dans les affiches des manuels d'Histoire de $1^{e}$, mémoire professionnel inédit, Université sciences humaines et sociales-Lille 3-Charles de Gaulles, 2012.

25 Adèle Clapperton-Richard, "Les "découvreurs", les "défenseurs", les "chefs" et les "mamans" : figures historiques dans l'enseignement de l'histoire nationale (19541976) ", communication présentée au Congrès de l'Institut d'histoire de l'Amérique française, Montréal, 20 octobre 2017.

26 Rose Fine-Meyer. «Including Women : The Establishment and Integration of Canadian Women's History into Toronto Ontario Classrooms 1968-1993 ", thèse de doctorat, Université de Toronto, 2012.

27 Pour une synthèse historique des changements aux programmes d'histoire québécois, voir : Félix Bouvier, Michel Allard, Paul Aubin et Marie-Claude Larouche, L'histoire nationale à l'école québécoise (Québec : Septentrion, 2012). 
28 Sabrina Moisan, Marie-Hélène Brunet et Audrey St-Onge, "Le récit du féminisme "à l'occidental" : enjeux pour l'apprentissage de l'histoire. Observations dans des classes de deuxième secondaire au Québec ", Revue Éducation, numéro thématique " genre et récit » à paraître.

29 Cicely Scheiner-Fisher et William Russell, «The Inclusion of Women's History in the Secondary Social Studies Classroom ", International Journal of Historical Learning, Teaching and Research 13, no. 1 (2015) : 97-117.

30 Linda Levstik et Jeanette Groth " "Scary Thing, Being An Eighth Grader" : Exploring Gender and Sexuality in a Middle School U.S. History Unit ", Theory and Research in Social Education 30, no. 2 (2002) : 240.

31 Keith Barton, "Agency, Choice and Historical Action : How History Teaching can Help Students Think about Democratic Decision Making ", communication présentée à la American Educational Research Association, New Orleans, 2011, 7.

32 Sur les documents iconographiques, voir Lauren Colley, "Taking the Stairs" to Break the Ceiling : Understanding Students' Conceptions of the Intersections of Historical Agency, Gender Equity and Action, thèse de doctorat, University of Kentucky, 2015. Sur les manuels, voir Marie-Hélène Brunet, Le féminisme dans les manuels d'histoire et d'éducation à la citoyenneté. Enquête auprès d'élèves québécois de quatrième secondaire, thèse de doctorat, Université de Montréal, 2016. 